\title{
Simultaneous Linearization of Holomorphic Maps with Hyperbolic and Parabolic Fixed Points
}

\author{
By \\ Tetsuo UEDA*
}

\begin{abstract}
We study local holomorphic mappings of one complex variable with parabolic fixed points as a limit of a families of mappings with attracting fixed points. We show that the Fatou coordinate for a parabolic fixed point can be obtained as a limit of some linear function of the solutions to Schröder equation for perturbed mappings with attracting fixed points.
\end{abstract}

\section{$\S 1 . \quad$ Introduction}

Let $g(w)$ be a holomorphic function of one variable of the form

$$
g(w)=\lambda w+\sum_{\nu=2}^{\infty} b_{\nu} w^{\nu}
$$

defined in a neighborhood of the origin 0 . If $0<|\lambda|<1$, then there are a neighborhood $V$ of 0 such that $g(V) \subset V$ and an injective holomorphic function $\rho(w)$ on $V$ satisfying the Schröder equation

$$
\rho(g(w))=\lambda \rho(w) .
$$

If $\lambda=1$ and $b_{2} \neq 0$, then there are a domain $V$ whose boundary contains 0 and an injective function $\varphi(w)$ (Fatou coordinate) satisfying the Abel equation

$$
\varphi(g(w))=\varphi(w)+1,
$$

\footnotetext{
Communicated by K. Saito. Received October 11, 2006. Revised June 15, 2007.

2000 Mathematics Subject Classification(s): 30D05, 37F45

This work is partially supported by Grant-in-Aid for Scientific Research (no. 15340055

and no. 17654031), Japan Society for the Promotion of Science.

*Department of Mathematics, Kyoto University, Kyoto 606-8502, Japan.

e-mail: ueda@math.kyoto-u.ac.jp
}

(c) 2008 Research Institute for Mathematical Sciences, Kyoto University. All rights reserved. 
which is unique up to an additive constant. (See Schröder [9], Koenigs [5], Leau [6], Fatou [3] and Milnor [8], for these classical results.)

In this paper, we consider families of functions $g_{\lambda}(w)$ which have $\lambda$ as a parameter and show that, when $\lambda$ tends non-tangentially to 1 from inside of the unit disk, some linear function of $\rho_{\lambda}(w)$ converges to $\varphi(w)$.

To do this it is convenient to consider the case where the fixed point is $\infty$ on the Riemann sphere. By scaling the coordinate, we consider a family of holomorphic maps of the form

$$
f_{\tau}(z)=\tau z+1+\frac{a_{1, \tau}}{z}+\frac{a_{2, \tau}}{z^{2}}+\cdots
$$

defined in a neighborhood of $\infty$. Here the parameter $\tau=1 / \lambda$ varies in a neighborhood of $\tau=1$. For $|\tau|>1$, let $\chi_{\tau}(z)$ denote the unique solution of the equation

$$
\chi_{\tau}(f(z))=\tau \chi_{\tau}(z)
$$

with $\chi_{\tau}(\infty)=\infty$ and normalized so that $\chi(z)=z+O(1)$ in a neighborhood of $\infty$. We will show that, when $\tau$ tends to 1 non-tangentially within the domain $|\tau|>1$, the sequence

$$
\chi_{\tau}(z)-\frac{1}{\tau-1}-a_{1, \tau} \log (\tau-1)
$$

converges to a solution to the Abel equation

$$
\varphi\left(f_{1}(z)\right)=\varphi(z)+1 .
$$

Precise statement and different formulations of the results are given in Theorems 3.3-3.6.

An alternative proof and a generalization is given recently by T. Kawahira [4]. As a related result, we note that T. C. McMullen showed the existence of quasiconformal maps giving conjugacies between $f_{\tau}$ and linear maps (see [7], Theorem 8.2).

\section{§2. Preliminaries}

\section{$\S 2.1$. A family of linear maps}

We begin with studying the family $\left\{\ell_{\tau}\right\}_{\tau}$ of linear maps

$$
\ell_{\tau}(z)=\tau z+1
$$

on the Riemann sphere $\widehat{\mathbb{C}}$ depending on the complex parameter $\tau$. When $|\tau|>$ 1 , the map $\ell_{\tau}$ has $\infty$ as an attracting fixed point and all points except for 
$1 /(1-\tau)$ converge locally uniformly to $\infty$ by the iterates of $\ell_{\tau}$. When $\tau=1$, then $\infty$ is a parabolic fixed point and all points in $\widehat{\mathbb{C}}$ converges to $\infty$, though the convergence is not uniform in the neighborhood of $\infty$.

We will investigate the uniformity, with respect to the parameter $\tau$, of the convergence of the iterates $\ell_{\tau}^{n}$, when $\tau$ tends to 1 non-tangentially from outside of the unit disk. So we will restrict the parameter $\tau$ in the closed sector

$$
T_{\alpha}=\{\tau \in \mathbb{C}|\operatorname{Re} \tau-1 \geq| \tau-1 \mid \cos \alpha\},
$$

where $\alpha$ is a real number with $0<\alpha<\pi / 2$, fixed throughout this paper.

To measure the rate of convergence to $\infty$, we introduce the function $N$ : $\widehat{\mathbb{C}} \times T_{\alpha}-\{(\infty, 1)\} \rightarrow \mathbb{R} \cup\{\infty\}$ as follows.

$$
\begin{array}{ll}
N_{\tau}(z)=\left|z-\frac{1}{1-\tau}\right|-\left|\frac{1}{1-\tau}\right| & \text { for }(z, \tau) \in \widehat{\mathbb{C}} \times\left(T_{\alpha}-\{1\}\right) ; \\
N_{1}(z)=\sup _{|\theta| \leq \alpha} \operatorname{Re}\left(e^{i \theta} z\right) & \text { for } z \in \mathbb{C} .
\end{array}
$$

We will not define $N_{1}(\infty)$. It is easy to see that the inequality

$$
\left|N_{\tau}\left(z_{1}\right)-N_{\tau}\left(z_{2}\right)\right| \leq\left|z_{1}-z_{2}\right| \quad z_{1}, z_{2} \in \mathbb{C}, \tau \in T_{\alpha}
$$

holds. In particular we have

$$
N_{\tau}(z) \leq|z|, \quad z \in \mathbb{C}, \tau \in T_{\alpha} .
$$

Lemma 2.1. $\quad N_{\tau}(z)$ is upper semi-continuous as a function of two variables $(z, \tau) \in \mathbb{C} \times T_{\alpha}-\{z=\infty\}$ and

$$
N_{1}(z)=\limsup _{T_{\alpha} \ni \tau \rightarrow 1} N_{\tau}(z) .
$$

Proof. For $r>0$, we let $\hat{N}_{(r, \theta)}(z)=N_{1+r e^{i \theta}}(z)$. Then

$$
\hat{N}_{(r, \theta)}(z)=\left|z+\frac{1}{r e^{i \theta}}\right|-\frac{1}{r}=\frac{1}{r}\left\{\left(1+2 r \operatorname{Re}\left(e^{i \theta} z\right)+r^{2}|z|^{2}\right)^{1 / 2}-1\right\} .
$$

This can be extended to a continuous function on $\widehat{\mathbb{C}} \times\{r \geq 0\} \times \mathbb{R}$, by defining $\hat{N}_{(0, \theta)}(z)=\operatorname{Re}\left(e^{i \theta} z\right)$. Hence

$$
\limsup _{T_{\alpha} \ni \tau \rightarrow 1} N_{\tau}(z)=\sup _{|\theta| \leq \alpha} \hat{N}_{(0, \theta)}(z)=\sup _{|\theta| \leq \alpha} \operatorname{Re}\left(e^{i \theta} z\right)=N_{1}(z) .
$$

This shows the assertion.

To have a uniform estimate of the rate of convergence of the iterats of $\ell_{\tau}$, let us first show the following: 
Lemma 2.2. For $(z, \tau) \in \widehat{\mathbb{C}} \times T_{\alpha}-\{(\infty, 1)\}$, we have

$$
N_{\tau}\left(\ell_{\tau}(z)\right) \geq|\tau| N_{\tau}(z)+\cos \alpha .
$$

Proof. First, if $\tau \in T-\{1\}$, then (1) is rewritten as

$$
\ell_{\tau}(z)-\frac{1}{1-\tau}=\tau\left(z-\frac{1}{1-\tau}\right)
$$

Hence

$$
\begin{aligned}
N_{\tau}\left(\ell_{\tau}(z)\right) & =\left|\ell_{\tau}(z)-\frac{1}{1-\tau}\right|-\left|\frac{1}{1-\tau}\right| \\
& =|\tau|\left|z-\frac{1}{1-\tau}\right|-\left|\frac{1}{1-\tau}\right| \\
& =|\tau| N_{\tau}(z)+\frac{|\tau|-1}{|1-\tau|} \\
& \geq|\tau| N_{\tau}(z)+\cos \alpha .
\end{aligned}
$$

Here we have used the fact that

$$
\frac{|\tau|-1}{|\tau-1|} \geq \frac{\operatorname{Re}(\tau)-1}{|\tau-1|} \geq \cos \alpha .
$$

If $\tau=1$, then $\ell_{1}(z)=z+1$, and hence

$$
\operatorname{Re}\left(e^{i \theta} \ell_{1}(z)\right)=\operatorname{Re}\left(e^{i \theta} z\right)+\cos \theta \geq \operatorname{Re}\left(e^{i \theta} z\right)+\cos \alpha .
$$

Therefore

$$
N_{1}\left(\ell_{1}(z)\right) \geq N_{1}(z)+\cos \alpha
$$

and the lemma is proved.

Let $R$ be a real number and define

$$
\mathcal{V}_{\alpha}(R)=\left\{(z, \tau) \in \widehat{\mathbb{C}} \times T_{\alpha}-\{(\infty, 1)\} \mid N_{\tau}(z)>R\right\} .
$$

We note that $\mathcal{V}_{\alpha}(R)$ is not open. Slices of $\mathcal{V}_{\alpha}(R)$ by $\tau=$ const. are open sets given by

$$
\begin{aligned}
& V_{\tau}(R)=\left\{z \in \widehat{\mathbb{C}} \mid N_{\tau}(z)>R\right\} \quad(\tau \neq 1) \\
& V_{1}(R)=\left\{z \in \mathbb{C} \mid N_{1}(z)>R\right\}=\bigcup_{|\theta| \leq \alpha}\left\{\operatorname{Re}\left(e^{i \theta} z\right)>R\right\} .
\end{aligned}
$$


Proposition 2.3. We have

$$
\left|\ell_{\tau}^{n}(z)\right| \geq n \cos \alpha \quad \text { for } \mathcal{V}_{\alpha}(0)
$$

and hence the sequence $\left\{\ell_{\tau}^{n}(z)\right\}_{n}$ converges to $\infty$ uniformly $\mathcal{V}_{\alpha}(0)$.

Proof. $\quad N_{\tau}(z)>0$ implies $N_{\tau}(\ell(z)) \geq N_{\tau}(z)+\cos \alpha>0$ by Lemma 2.2. Hence, if $\tau \in T_{\alpha}$ and $z \in V_{\tau}(0)$, then $\ell_{\tau}^{n}(z) \in V_{\tau}(0)$ and

$$
\left|\ell_{\tau}^{n}(z)\right| \geq N_{\tau}\left(\ell_{\tau}^{n}(z)\right) \geq N_{\tau}(z)+n \cos \alpha \geq n \cos \alpha,
$$

for all $n$. This proves the assertion.

\section{§2.2. Solution to a difference equation}

We consider the difference equation

$$
h_{\tau}\left(\ell_{\tau}(z)\right)-\tau h_{\tau}(z)=\frac{1}{z}+C_{\tau},
$$

where $\ell_{\tau}(z)=\tau z+1$ with $|\tau|>1$ or $\tau=1$; and $C_{\tau}$ is a constant depending on $\tau$, which will be given later.

A solution to this equation is given by

$$
h_{\tau}(z)=-\frac{1}{\tau z}+\sum_{n=1}^{\infty} \frac{1}{\tau^{n+1}}\left\{\frac{1}{\ell_{\tau}^{n}(0)}-\frac{1}{\ell_{\tau}^{n}(z)}\right\} .
$$

We note that $\ell_{\tau}(z)=\tau^{n} z+\tau^{n-1}+\cdots+\tau+1$ and $\ell_{\tau}(0)=\tau^{n-1}+\cdots+\tau+1$. In the following, we will investigate some properties of this function.

First, for a $\tau$ fixed, the following properties of $h_{\tau}(z)$ can be easily verified: In the case $|\tau|>1$, the function $h_{\tau}(z)$ is meromorphic on $\widehat{\mathbb{C}}$ except the essential singularity at $1 /(1-\tau)$, and has poles at $\left(1-\tau^{-n}\right) /(1-\tau),(n=0,1,2, \ldots)$. This function $h_{\tau}(z)$ is holomorphic at $\infty$. We write

$$
H_{\tau}=h_{\tau}(\infty)=\sum_{n=1}^{\infty} \frac{1}{\tau^{n+1} \ell_{\tau}^{n}(0)} .
$$

We can easily verify that $h_{\tau}(z)$, with $\tau \neq 1$, satisfies the equation (3) with the constant

$$
C_{\tau}=(1-\tau) H_{\tau} .
$$

In the case $\tau=1$, we have $\ell^{n}(z)=z+n$ and

$$
h_{1}(z)=-\frac{1}{z}+\sum_{n=1}^{\infty}\left\{\frac{1}{n}-\frac{1}{z+n}\right\} \text {. }
$$


This function is meromorphic on $\mathbb{C}$ and has poles at $0,-1,-2, \ldots$ As is easily verified, $h_{1}(z)$ satisfies the equation (3) with $C_{1}=0$.

We note that

$$
h_{1}(z)=\frac{\Gamma^{\prime}(z)}{\Gamma(z)}+\gamma
$$

where $\Gamma(z)$ denotes the gamma function and $\gamma$ denotes the Euler constant

$$
\gamma=\lim _{n \rightarrow \infty}\left(\sum_{k=1}^{n} \frac{1}{k}-\log n\right)
$$

Now we study the dependence of $h_{\tau}(z)$ on the parameter $\tau$.

Proposition 2.4. The function $h_{\tau}(z)$ is continuous on $\mathcal{V}_{\alpha}(0)$.

Proof. The continuity at the points $(z, \tau)$ with $\tau \neq 1$ is clear. Using $\ell_{\tau}^{n}(z)-\ell_{\tau}^{n}(0)=\tau^{n} z$ and the estimate (2), we have

$$
\left|\frac{1}{\tau^{n+1}}\left\{\frac{1}{\ell_{\tau}^{n}(0)}-\frac{1}{\ell_{\tau}^{n}(z)}\right\}\right|=\left|\frac{z}{\tau \ell_{\tau}^{n}(0) \ell_{\tau}^{n}(z)}\right| \leq\left|\frac{z}{\tau}\right| \frac{1}{n^{2} \cos ^{2} \alpha} .
$$

This shows that the series (4) is locally uniformly convergent on $\mathcal{V}_{\alpha}(0)-$ $\{z=\infty\}$ and hence $h_{\tau}(z)$ is continuous there.

Corollary 2.5. The constat $C_{\tau}$ is a continuous function of $\tau \in T_{\alpha}$.

Proof. By the difference equation (3), we have $C_{\tau}=h_{\tau}\left(\ell_{\tau}(z)\right)-\tau h_{\tau}(z)-$ $1 / z$, which is continuous on $\mathcal{V}_{\alpha}(0)$ by Proposition 2.4. Hence $C_{\tau}$ is continuous on $T_{\alpha}$.

Proposition 2.6. For any $\varepsilon>0$, there is a constant $M$ such that

$$
\left|h_{\tau}^{\prime}(z)\right| \leq \frac{M}{N_{\tau}(z)} \quad \text { on } \mathcal{V}_{\alpha}(\varepsilon) .
$$

Proof. Differentiation of (3) with respect to $z$ yields

$$
h_{\tau}^{\prime}(z)=\frac{1}{\tau} \sum_{n=0}^{\infty} \frac{1}{\left\{\ell_{\tau}^{n}(z)\right\}^{2}} .
$$

Hence

$$
\begin{aligned}
\left|h_{\tau}^{\prime}(z)\right| \leq \sum_{n=0}^{\infty} \frac{1}{\left|\ell_{\tau}^{n}(z)\right|^{2}} & \leq \sum_{n=0}^{\infty} \frac{1}{\left(N_{\tau}(z)+n \cos \theta\right)^{2}} \\
& \leq \int_{0}^{\infty} \frac{d x}{\left(N_{r}(z)+x \cos \theta\right)^{2}}
\end{aligned}
$$

Therefore $\left|h_{\tau}^{\prime}(z)\right|$ is bounded by $M / N_{\tau}(z)$ with some constant $M$. 


\section{§2.3. Behavior of $H_{\tau}$}

Now we look at the behavior of the function $H_{\tau}$ defined by (5), when $\tau \rightarrow 1$ within the sector $T$. It is clear from the expression (5) that $H_{\tau}$ is unbounded, while $C_{\tau}=(1-\tau) H_{\tau}$ tends to 0 by Corollary 2.5. Here we give a more precise description of its behavior.

Proposition 2.7. We have

$$
H_{\tau}=-\log (\tau-1)+\gamma-1+o(1)
$$

as $\tau \rightarrow 1$ within the sector $T_{\alpha}$. Here $\gamma$ denotes the Euler constant.

Proof. To begin with, letting $\lambda=1 / \tau$, we have

$$
\begin{aligned}
H_{1 / \lambda} & =\sum_{n=1}^{\infty} \frac{\lambda^{2 n}}{1+\lambda+\cdots+\lambda^{n-1}} \\
& =(1-\lambda) \sum_{n=1}^{\infty}\left(\frac{\lambda^{n}}{1-\lambda^{n}}-\lambda^{n}\right) \\
& =(1-\lambda) L(\lambda)-\lambda .
\end{aligned}
$$

Here $L(\lambda)$ denotes the Lambert series defined by

$$
L(\lambda)=\sum_{n=1}^{\infty} \frac{\lambda^{n}}{1-\lambda^{n}} .
$$

This series $L(\lambda)$ defines a holomorphic function on $|\lambda|<1$. We want to know the behavior of this function when $\lambda$ tends to 1 non-tangentially within the unit disk.

$L(\lambda)$ is developped into the power series

$$
L(\lambda)=\sum_{n=1}^{\infty} d(n) \lambda^{n}=\lambda+2 \lambda^{2}+2 \lambda^{3}+3 \lambda^{4}+\cdots,
$$

where $d(n)$ denotes the number of divisors of $n$. We write

$$
D(n)=d(1)+\cdots+d(n) .
$$

Then

$$
\frac{L(\lambda)}{1-\lambda}=\sum_{n=1}^{\infty} D(n) \lambda^{n}
$$


The asymptotic behavior of $D(n)$ is given by a theorem of Dirichlet (see Apostol [1], Chandrasekharan [2]) :

$$
D(n)=n \log n+(2 \gamma-1) n+O(\sqrt{n}) \quad(n \rightarrow \infty)
$$

From this and the fact that

$$
\sum_{k=1}^{n} \frac{1}{k}-\log n=\gamma+O\left(\frac{1}{n}\right)
$$

it follows that

$$
\begin{aligned}
D(n) & =n \sum_{k=1}^{n} \frac{1}{k}+(\gamma-1) n+p_{n} \\
& =\sum_{k=1}^{n} \frac{n-k}{k}+\gamma n+p_{n}
\end{aligned}
$$

where $p_{n}=O(\sqrt{n})$ as $n \rightarrow \infty$. Therefore, noting that

$$
\frac{\lambda}{(1-\lambda)^{2}}=\sum_{n=1}^{\infty} n \lambda^{n}, \quad \log (1-\lambda)=-\sum_{n=1}^{\infty} \frac{\lambda^{n}}{n}
$$

we have

$$
\frac{L(\lambda)}{1-\lambda}=\sum_{n=1}^{\infty} D(n) \lambda^{n}=-\frac{\lambda \log (1-\lambda)}{(1-\lambda)^{2}}+\frac{\gamma \lambda}{(1-\lambda)^{2}}+P(\lambda)
$$

where $P(\lambda)=\sum_{n=1}^{\infty} p_{n} \lambda^{n}$. Since $p_{n}=O(\sqrt{n})=o(n)$, we have

$$
P(\lambda)=o\left((1-\lambda)^{-2}\right) \quad \text { as } \lambda \rightarrow 1 \text { non-tangentially. }
$$

Thus we obtain

$$
\begin{aligned}
H_{\tau} & =(1-\lambda) L(\lambda)-\lambda \\
& =-\lambda \log (1-\lambda)+(\gamma-1) \lambda+(1-\lambda)^{2} P(\lambda) \\
& =-\log (\tau-1)+\gamma-1+o(\tau-1)
\end{aligned}
$$

and the proposition is proved. 


\section{§3. Families of Maps with Attracting/Parabolic Fixed Points}

\section{§3.1. Domain of convergence}

Let $U(R)=\{z \in \widehat{\mathbb{C}}|R<| z \mid \leq \infty\}$ be a neighborhood of $\infty \in \widehat{\mathbb{C}}$ and we consider a family of holomorphic maps $f_{\tau}: U(R) \rightarrow \widehat{\mathbb{C}}$ of the form

$$
f_{\tau}(z)=\tau z+1+A_{\tau}(z)
$$

with

$$
A_{\tau}(z)=\frac{a_{1, \tau}}{z}+\frac{a_{2, \tau}}{z^{2}}+\cdots .
$$

We suppose that $f_{\tau}$ depends holomorphically on the parameter $\tau \in \Delta_{\rho}=\{\tau \in$ $\mathbb{C}|| \tau-1 \mid<\rho\}$.

As in the previous section, we choose and fix $\alpha$ so that $0<\alpha<\pi / 2$ and let $\delta=\frac{1}{2} \cos \alpha$. By shrinking the neighbohoods $U(R)$ and $\Delta_{\rho}$, we assume that there is a constant $K$ such

$$
\left|A_{\tau}(z)\right|<\frac{K}{|z|}<\delta
$$

for $(z, \tau) \in U(R) \times \Delta_{\rho}$. Further we assume that $f_{\tau}(z)$ is injective in $z$ for every $\tau \in \Delta_{\rho}$.

Now we have results on uniformity of convergence for $f_{\tau}^{n}(z)$, corresponding to Lemma 2.2 and Proposition 2.3 for $\ell_{\tau}(z)$. We set

$$
T_{\alpha, \rho}=T_{\alpha} \cap \Delta_{\rho}=\{\tau \in \mathbb{C}|\operatorname{Re}(\tau-1) \leq| \tau-1|\cos \alpha,| \tau-1 \mid<\rho\} .
$$

Lemma 3.1. $\quad$ For $(z, \tau) \in U(R) \times T_{\alpha, \rho}-\{(\infty, 1)\}$ we have

$$
N_{\tau}\left(f_{\tau}(z)\right) \geq|\tau| N_{\tau}(z)+\delta .
$$

Proof. From $f_{\tau}(z)=\ell_{\tau}(z)+A_{\tau}(z)$, it follows that

$$
\begin{aligned}
N_{\tau}\left(f_{\tau}(z)\right) & \geq N_{\tau}\left(\ell_{\tau}(z)\right)-\left|A_{\tau}(z)\right| \\
& \geq|\tau| N_{\tau}(z)+\cos \alpha-\delta \\
& =|\tau| N_{\tau}(z)+\delta,
\end{aligned}
$$

which proves the lemma.

Now let

$$
\mathcal{V}_{\alpha, \rho}(R)=\left\{(z, \tau) \in \mathcal{V}_{\alpha}(R) \mid \tau \in T_{\alpha, \rho}\right\} .
$$

We note that $\mathcal{V}_{\alpha, \rho}(R) \subset U(R) \times T_{\alpha, \rho}$ since $N_{\tau}(z) \leq|z|$. 
Proposition 3.2. If $\tau \in T_{\alpha, \rho}$ and $z \in V_{\tau}(R)$, then $f_{\tau}(z) \in V_{\tau}(R)$. The sequence $\left\{f_{\tau}^{n}(z)\right\}_{n}$ converges uniformly on $\mathcal{V}_{\alpha, \rho}(R)$ to $\infty$.

Proof. If $\tau \in T_{\alpha, \rho}$ and $z \in V_{\tau}(R)$, then $N_{\tau}(z)>R$. Hence $N_{\tau}\left(f_{\tau}(z)\right) \geq$ $N_{\tau}(z)+\delta>R+\delta$ and $f_{\tau}(z) \in V_{\tau}(R)$. Further

$$
\left|f_{\tau}^{n}(z)\right| \geq N_{\tau}\left(f_{\tau}^{n}(z)\right) \geq N_{\tau}(z)+n \delta>R+n \delta .
$$

This shows the uniform convergence of $\left\{f_{\tau}^{n}(z)\right\}_{n}$ to $\infty$ on $\mathcal{V}_{\alpha, \rho}(R)$.

\section{§3.2. Schröder-Abel equation}

We recall that $C_{\tau}$ is continuous on $T_{\alpha}$ and holomorphic in the interior of $T_{\alpha}$ and that $C_{\tau}=(1-\tau) H_{\tau}$ when $\tau \neq 1$. Let

$$
B_{\tau}=1-a_{1, \tau} C_{\tau} .
$$

The following theorem constitutes the main ingredient of this paper.

Theorem 3.3. $\quad$ There exists a function $\varphi_{\tau}(z)$ on $\mathcal{V}_{\alpha, \rho}(R)$ with values in $\widehat{\mathbb{C}}$ satisfying the following conditions:

(i) $\varphi_{\tau}(z)$ is continuous on $\mathcal{V}_{\alpha, \rho}(R)$ and holomorphic in its interior as a mapping to $\widehat{\mathbb{C}}$.

(ii) For each $\tau \in T_{\alpha, \rho}-\{1\}$ fixed, the function $\varphi_{\tau}(z)$ is holomorphic in $V_{\tau}(R)$ except for a simple pole at $\infty$; and $\varphi_{1}(z)$ is holomorphic in $V_{1}(R)$. Further $\varphi_{\tau}(z)$ satisfies the functional equation

$$
\varphi_{\tau}\left(f_{\tau}(z)\right)=\tau \varphi_{\tau}(z)+B_{\tau} .
$$

(iii) For each $\tau \in T_{\alpha, \rho}-\{1\}$ fixed, the function $\varphi_{\tau}(z)$ is of the form

$$
\varphi_{\tau}(z)=z-a_{1, \tau} H_{\tau}+o(1)
$$

in a neighborhood of $z=\infty$.

The proof is given in the next subsection.

This theorem implies in particular the following: Suppose that $\tau$ tends to 1 from outside of the unit disk with direction $\theta$, i.e., $\tau=1+r e^{i \theta}$ which fixed $\theta$ and $r$ tending to 0 . Then the domain $V_{\tau}(R)$ of $\varphi_{\tau}(z)$ converges to the half plane $\left\{\operatorname{Re} e^{i \theta} z>R\right\} \subset V_{1}(R)$, and $\varphi_{\tau}(z)$ converges to $\varphi_{1}(z)$ on this half plane. This remark applies also to $\psi_{\tau}$ given below.

To make clear the meaning of this theorem, we will give the relation between $\varphi_{\tau}(z)$ and the solution to Schröder equation. 
Suppose $|\tau|>1$ and consider the equation

$$
\chi_{\tau}\left(f_{\tau}(z)\right)=\tau \chi_{\tau}(z)
$$

which is a variant of the Schröder equation formutated for the case where the fixed point is $\infty$. It is classical that this equation has a unique solution $\chi_{\tau}(z)$ of the form $\chi_{\tau}(z)=z+O(1)$ in a neighborhood of $\infty$. By comparing the coefficients of the Laurent expansion we can see that

$$
\chi_{\tau}(z)=z+\frac{1}{\tau-1}+O(1 / z)
$$

On the other hand, we can easily verify that $\varphi(z)+B_{\tau} /(\tau-1)$ satisfies the equation (11). Since $B_{\tau}=1-a_{1, \tau} C_{\tau}=1-a_{1, \tau}(1-\tau) H_{\tau}$ by (9) and (6), we have the following.

Theorem 3.4. For $\tau \in T_{\alpha, \rho}-\{1\}$ we have

$$
\begin{aligned}
\varphi_{\tau}(z) & =\chi_{\tau}(z)-\frac{B_{\tau}}{\tau-1} \\
& =\chi_{\tau}(z)-\frac{1}{\tau-1}-a_{1, \tau} H_{\tau} .
\end{aligned}
$$

Our result may be stated, without referring to $\varphi_{\tau}(z)$, as follows.

Theorem 3.5. When $\tau$ tends to 1 non-tangentially from outside of the unit disk, the function

$$
\chi_{\tau}(z)-\frac{1}{\tau-1}+a_{1, \tau} \log (\tau-1)
$$

for $\tau \in T-\{1\}$ converges to a solution to the Abel equation for $f_{1}(z)$.

Here we may replace $a_{1, \tau}$ by $a_{1,1}$, since $a_{1, \tau} H_{\tau}$ and $-a_{1,1} \log (\tau-1)$ differ only by a continuous function on $T_{\alpha}, \rho$.

We may normalize $\varphi_{\tau}(z)$ by letting $\varphi_{\tau}^{*}(z)=\varphi_{\tau}(z) / B_{\tau}$. Then $\varphi_{\tau}^{*}(z)$ satisfies the conditions of Theorem 3.3, replacing $B_{\tau}$ by 1 . For $\tau \neq 1$, we have

$$
\varphi_{\tau}^{*}(z)=\frac{\chi_{\tau}(z)}{B_{\tau}}-\frac{1}{\tau-1} .
$$

Now we give another reformulations of the result. The function $\varphi_{\tau}$ has pole on $z=\infty$. By a linear fractional transformation, we obtain a function which is holomorphic on $\mathcal{V}_{\alpha, \rho}(R)$. 
Theorem 3.6. There exists a function $\psi_{\tau}(z)$ on $\mathcal{V}_{\alpha, \rho}(R)$ satisfying the following conditions:

(i) $\psi_{\tau}(z)$ is continuous on $\mathcal{V}_{\alpha, \rho}(R)$ and holomorphic in its interior, as a function of two variables.

(ii) For each $\tau \in T_{\alpha, \rho}$ fixed, the function $\psi_{\tau}(z)$ is holomorphic in $V_{\tau}(R)$ and satisfies the functional equation

$$
\psi_{\tau}\left(f_{\tau}(z)\right)=\frac{1}{\tau} \psi_{\tau}(z)+1
$$

(iii) For each $\tau \in T_{\alpha, \rho}$ fixed, the function $\psi_{\tau}(z)$ is of the form

$$
\psi_{\tau}(z)=\frac{\tau}{\tau-1}-\frac{\tau B_{\tau}}{(\tau-1)^{2}} \frac{1}{z}+O\left(\frac{1}{z^{2}}\right) .
$$

In the neighborhood of $z=\infty$,

Proof. We define

$$
\psi_{\tau}(z)=\frac{\tau \varphi_{\tau}(z)}{(\tau-1) \varphi_{\tau}(z)+B_{\tau}} .
$$

Then

$$
\begin{aligned}
& \psi_{\tau}(z)=\frac{\tau}{\tau-1}-\frac{\tau B_{\tau}}{(\tau-1)^{2}} \frac{1}{\chi_{\tau}(z)} \quad \text { when } \tau \neq 1 . \\
& \psi_{1}(z)=\varphi_{1}^{*}(z) .
\end{aligned}
$$

We can easily verify that $\psi_{\tau}(z)$ satisfies the required conditions.

\section{§3.3. $\quad$ Proof of Theorem 3.3}

To simplify the notation, we omit the subscript $\tau$ for $f_{\tau}$ etc.

We rewrite the expression (7) in the form

$$
f(z)=\tau z+1+\frac{a_{1, \tau}}{z}+A_{1}(z)
$$

with $A_{1, \tau}(z)=a_{2}(\tau) / z^{2}+\ldots$ There exits some constant $K_{1}$ such that

$$
\left|A_{1}(z)\right| \leq \frac{K_{1}}{|z|^{2}}
$$

To make clear the idea of the proof, we will first consider the case where $a_{1, \tau}=0$ identically. Replacing $z$ by $f^{n-1}(z)$ for in (11) and dividing by $\tau^{n}$, we obtain

$$
\frac{1}{\tau^{n}} f^{n}(z)=\frac{1}{\tau^{n-1}} f^{n-1}(z)+\frac{1}{\tau^{n}}+\frac{1}{\tau^{n}} A_{1}\left(f^{n-1}(z)\right)
$$


We define

$$
\varphi_{n}(z):=\frac{1}{\tau^{n}} f^{n}(z)-\sum_{k=1}^{n} \frac{1}{\tau^{k}}=z+\sum_{k=1}^{n} \frac{1}{\tau^{k}} A_{1}\left(f^{k-1}(z)\right) .
$$

Since

(14) $\left|\frac{1}{\tau^{k}} A_{1}\left(f^{n-1}(p)\right)\right| \leq \frac{K_{1}}{\left|f^{n-1}(z)\right|^{2}} \leq \frac{K_{1}}{N\left(f^{n-1}(z)\right)^{2}} \leq \frac{K_{1}}{(N(z)+(n-1) \delta)^{2}}$,

we conclude that $\varphi_{n}(z)$ converges uniformly as $n \rightarrow \infty$. Therefore the limit $\varphi(z):=\lim _{n \rightarrow \infty} \varphi_{n}(z)$ is continuous on $\mathcal{V}_{\alpha, \rho}(R)$. From $\varphi_{n}(f(z))=\tau \varphi_{n+1}(z)+$ 1 , it follows that $\varphi(z)$ satisfies the equation (10) with $B_{\tau}=1$.

Now, in the general case where $a_{1, \tau}$ does not vanish identically, we have to modify the above construction to have convergent sequence. Let us recall the function $h(z)$ satisfying the difference equation (3) in the previous section. We set

$$
A_{2}(z)=h(f(z))-h(\ell(z))
$$

Then

$$
h(f(z))=\tau h(z)+C_{\tau}+\frac{1}{z}+A_{2}(z) .
$$

Combining this with (12), we get

$$
f(z)-a_{1} h(f(z))=\tau\left\{z-a_{1} h(z)\right\}+B_{\tau}+\tilde{A}(z)
$$

with $B_{\tau}=1-a_{1} C_{\tau}$, where we have set

$$
\tilde{A}(z)=A_{1}(z)-a_{1} A_{2}(z)
$$

In the same manner as in (13), we obtain

$\frac{1}{\tau^{n}}\left\{f^{n}(z)-a_{1} h\left(f^{n}(z)\right)\right\}=\frac{1}{\tau^{n-1}}\left\{f^{n-1}(z)-a_{1} h\left(f^{n-1}(z)\right)\right\}+\frac{B_{\tau}}{\tau^{n}}+\frac{1}{\tau^{n}} \tilde{A}\left(f^{n-1}(z)\right)$.

We define

$$
\begin{aligned}
\varphi_{n}(z) & =\frac{1}{\tau^{n}}\left\{f^{n}(z)-a_{1} h\left(f^{n}(z)\right)\right\}-B_{\tau} \sum_{k=1}^{n} \frac{1}{\tau^{k}} \\
& =z-a_{1} h(z)+\sum_{k=1}^{n} \frac{1}{\tau^{k}} \tilde{A}\left(f^{k-1}(z)\right) .
\end{aligned}
$$

The sum on the right is

$$
\sum_{k=1}^{n} \frac{1}{\tau^{k}} A_{1}\left(f^{k-1}(z)\right)-a_{1, \tau} \sum_{k=1}^{n} \frac{1}{\tau^{k}} A_{2}\left(f^{k-1}(z)\right) .
$$


When $n \rightarrow \infty$, the first sum is uniformly convergent by the estimate (14). The convergence of the second sum follows from Lemma 3.7 below. Thus $\varphi_{n}(z)$ converges uniformly on $\mathcal{V}_{\alpha, \rho}(R)$ as $n \rightarrow \infty$. Hence the limit $\varphi(z)=$ $\lim _{n \rightarrow \infty} \varphi_{n}(z)$ is continuous on $\mathcal{V}_{\alpha, \rho}(R)$. From $\varphi_{n}(f(z))=\tau \varphi_{n+1}(z)+B_{\tau}$ it follows that $\varphi(z)$ satisfies the equation (10).

Since $\tilde{A}(z)$ vanishes at $z=\infty$, we have $\varphi_{n}(z)=z-a_{1} H_{\tau}+o(1)$ in the neighborhood of $z=\infty$. Letting $n \rightarrow \infty$ yields the assertion (iii).

Lemma 3.7. We have

$$
\left|A_{2}(z)\right| \leq \frac{K M}{|z| N(z)}
$$

on $(z, \tau) \in \mathcal{V}_{\alpha, \rho}(R)$.

Proof. Let $z$ be a point with $N(z)>R$ and let $[\ell(z), f(z)]$ denote the segment joining $\ell(z)$ and $f(z)$ in $\mathbb{C}$. The length of this segment is

$$
|f(z)-\ell(z)|=|A(z)|<\frac{K}{|z|}<\delta,
$$

by (8). For any $\zeta$ in this segment, we have $|N(\zeta)-N(\ell(z))| \leq|\zeta-\ell(z)|<\delta$. Hence $N(\zeta)>N(\ell(z))-\delta>N(z)$. Hence, by Proposition 2.6 we have

$$
\left|h^{\prime}(\zeta)\right| \leq \frac{M}{N(\zeta)} \leq \frac{M}{N(z)}
$$

on this segment. Thus we have

$$
\left|A_{2}(z)\right|=\left|\int_{[\ell(z), f(z)]} h^{\prime}(\zeta) d \zeta\right| \leq \frac{K M}{|z| N(z)},
$$

which proves the assertion.

\section{Acknowledgement}

The author would like to thank the referee for careful reading of the manuscript and helpful suggestions.

\section{References}

[1] T. M. Apostol, Introduction to analytic number theory, Springer, New York, 1976. 
[2] K. Chandrasekharan, Introduction to analytic number theory, Springer-Verlag New York Inc., New York, 1968.

[3] P. Fatou, Sur les équations fonctionelles, Bull. Soc. Math. France 47 (1919) 161-271; ibid. 48 (1920) 33-94, 208-314.

[4] T. Kawahira, A proof of simultaneous linearization with a polylog estimate, Bull. Pol. Acad. Sci. Math. 55 (2007), no. 1, 43-52.

[5] G. Koenigs, Recherches sur les integrals de certaines equations fonctionelles, Ann. Sci. Ec. Norm. Sup. (3e Ser.) 1 (1884) supplem. 1-41.

[6] L. Leau, Étude sur les équations fonctionnelles à une ou à plusieurs variables, Ann. Fac. Sci. Toulouse Sci. Math. Sci. Phys. 11 (1897), no. 2, E1-E24; ibid. no. 3, E25-E110.

[7] C. T. McMullen, Hausdorff dimension and conformal dynamics. II. Geometrically finite rational maps, Comment. Math. Helv. 75 (2000), no. 4, 535-593.

[8] J. Milnor, Dynamics in one complex variable, Third edition, Princeton Univ. Press, Princeton, NJ, 2006.

[9] E. Schröder, Ueber iterierte Funktionen, Math. Ann. 3 (1871), 296-322. 